\title{
Obesity and Mental Health: A Longitudinal, Cross-Cultural Examination in Germany and China
}

\author{
Kristen L. Lavallee, Xiao Chi Zhang, Silvia Schneider and Jürgen Margraf* \\ Mental Health Research and Treatment Center, Ruhr-Universität Bochum, Bochum, Germany
}

OPEN ACCESS

Edited by:

Thomas Kubiak,

Johannes Gutenberg University

Mainz, Germany

Reviewed by:

Norbert Hermanns,

Research Institute of the Diabetes

Academy Mergentheim (FIDAM),

Germany

Kathryn Anne Nel,

University of Limpopo,

South Africa

*Correspondence:

Jürgen Margraf

juergen.margraf@rub.de

Specialty section:

This article was submitted to

Eating Behavior,

a section of the journal

Frontiers in Psychology

Received: 20 May 2021 Accepted: 16 August 2021 Published: 21 September 2021

Citation:

Lavallee $K L$, Zhang $X C$,

Schneider S and Margraf J (2021)

Obesity and Mental Health: A

Longitudinal, Cross-Cultural

Examination in Germany and China.

Front. Psychol. 12:712567.

doi: 10.3389/fpsyg.2021.712567
The present study examines the relationship between obesity and mental health using longitudinal data. Participants with data at baseline and one-year follow-up were included from two countries: Germany (364) and China (9007). A series of structural equation models with three mediators and one moderator were conducted separately for female and male students in Germany and China. Zero-order correlations indicated that overweight/obesity was significantly related to later depression and anxiety in Chinese males. Additional effects of obesity on later mental health flowed through effects on attractiveness (Chinese and German females, and Chinese males), physical health (Chinese males), and life satisfaction (German females). Though overweight/obesity is related to mental health across many other studies, results in this study yield total effects between overweight/obesity and follow-up mental health only in Chinese males. The relationship between overweight/obesity and follow-up mental health was significantly mediated by follow-up attractiveness, or health state, or life satisfaction in German females, Chinese females, and Chinese male students, with no significant indirect effects found in German male students. This highlights the possible importance of culture in examining these effects.

Keywords: positive mental health, depression, anxiety, longitudinal, cross-cultural, negative mental health

\section{OBESITY AND MENTAL HEALTH: A LONGITUDINAL, CROSS- CULTURAL EXAMINATION}

A preponderance of high-calorie, low-nutrient, and processed food, combined with increasingly sedentary lifestyles, has catapulted obesity rates to all-time highs. Pediatric rates for the newest additions to the population emerging from toddlerhood, young children between ages 5-9, are now at $10.4 \%$ in France, $15.1 \%$ in Brazil, $17.5 \%$ in China and Mexico, $22.7 \%$ in the US, and as high as $36.3 \%$ in the Pacific Islands (NCD Risk Factor Collaboration, 2017), affecting approximately one in five young people globally (Baker et al., 2017). Recent years have seen an important cultural shift away from weight and size to focusing on health. This shift toward health and away from a focus on size has been effective at improving overall health outcomes, reducing stigma, and maintaining self-esteem (Bacon, 2010). Nevertheless, overall rates of obesity have continued to rise and the consequences of obesity on physical and mental health remain. Many physical ailments and conditions, including obesity, are associated with serious mental disorders (Iacovides and Siamouli, 2008), and in particular anxiety and depression. In people with obesity, feelings of shame, guilt, and social anxiety are common and can be particularly 
prevalent in those with Binge Eating Disorder, as they are directly related to the disorder (Albohn-Kuhne and Rief, 2011). However, obesity is also more common in those with mental health concerns not typically considered part of disordered eating, such as schizophrenia (Bradshaw and Mairs, 2014), and depression (Chauvet-Gelinier et al., 2019). Some weight gain is associated with medications to treat mental disorders (Daumit et al., 2003; Bradshaw and Mairs, 2014), but medications do not explain all of the effects (Allison et al., 2009; Holt and Peveler, 2009). Further, the relationship between obesity and mental disorder has been investigated primarily in Western populations, leaving cross-cultural differences underexplored. The relationship between obesity and positive mental health, or protective factors, if any, remains largely uncharted.

\section{Obesity and Mental Disorder}

Anxiety and obesity are the two most common health complaints and are often co-occurring. Overweight is associated with increased anxiety and depression with moderate effects (OR in the range of 1.2-1.5) across 13 different countries, concentrated in those with more severe obesity and in females in a largescale survey within the World Mental Health Surveys (Scott et al., 2008). Across 25 studies included in one large metaanalysis, the frequency of anxiety occurred at an odds ratio of 1.30 in those with obesity and 1.10 in those with overweight. Aggregated effects were similar across men and women in this meta-analysis (Amiri and Behnezhad, 2019). A slightly older meta-analysis found similar effect sizes across 16 studies (2 prospective, with the remaining 14 cross-sectional), with an odds ratio of 1.4 for the association between obesity and anxiety (Gariepy et al., 2010). Obesity is also related to mood disorders. In a community study of over 36,000 Canadians, lifetime major depression was related to concurrent obesity with an odds ratio of 1.46 in females, though not related to obesity in males in this study (McIntyre et al., 2006).

Longitudinal research indicates a bidirectional relationship between obesity and mental health. One 11-year longitudinal study of the effects of mental health on weight gain and obesity in over 25,000 Norwegian adults found that the presence of anxiety or depression at baseline predicted increased weight gain and increased incidence of initial obesity over time (Brumpton et al., 2013). A comprehensive meta-analysis of the longitudinal bidirectional relationship between obesity/ overweight and depression across 15 studies demonstrated that depression was predicted by both baseline obesity (odds ratio 1.55; stronger for Americans than Europeans) and baseline overweight (odds ratio 1.27). Further, baseline depression predicted later obesity (odds ratio 1.58), though not overweight. Moderators of these effects across studies in the meta-analysis, such as sex, age, and depression severity, were not significant, indicating that the link between depression and obesity was present regardless of sex, age, and severity of depression. The effects were significant for adults, but not teens in this metaanalysis (Luppino et al., 2010). Another more recent review found obesity linked to both mental distress and sexual dysfunction but found that causal links were difficult to draw from the existing research in the area of sexual dysfunction (Esfahani and Pal, 2018). While the Luppino meta-analysis did not find gender differences (Luppino et al., 2010), some individual studies have. Anxiety was a particular risk factor for later obesity in adolescent males in one study of youth aged 11-17 in the United States (Roberts and Duong, 2016). However, another study found significant longitudinal effects of obesity on increased risk for subsequent depression (adjusted hazard ratio, $H R=3.9)$ and anxiety disorder $(H R=3.8)$ for United States female adolescents, but not for males (Anderson et al., 2007). It may be that gender effects, while sometimes present in individual studies, average out to no effect when taken together across studies, or may become evident only in individual studies illuminating effects for teens, which Luppino did not find when examining effects across studies (Luppino et al., 2010).

Though the Luppino meta-analysis did not find effects for teens (Luppino et al., 2010), other research does point to some association between obesity and mental health in children and teens. For example, a large-scale population study of over 43,000 United States children found a depression rate of $15 \%$ for overweight, and $16 \%$ for obesity, with those classified as obese reporting increased internalizing (AOR 1.59) and externalizing (AOR 1.33) problems (Halfon et al., 2013). In a study of teens with severe mental illness, the teens were $30 \%$ likely to have obesity, and $55.5 \%$ likely to have overweight or obesity, rates approximately double national United States averages. Greater obesity in this study was related to lack of private health insurance (a stand-in for poverty), smoking, and antidepressant and antipsychotic medication use (Gracious et al., 2010). In another study of mental health in overweight and obese teens, the relationship between obesity and mental distress increased with severity of obesity (Calderon et al., 2009).

\section{Mechanisms Facilitating the Relationship Between Obesity and Mental Disorder}

The relationship between obesity and mental disorder, like most relationships, is multiply determined. A study comparing psychiatric outpatients to the general population found that, as expected, psychiatric outpatients are more likely to have obesity than those in the general population. However, obesity was not related to the presence of psychiatric illness in this study, but rather to other co-occurring demographic factors, such as older age, gender, and comorbid chronic illnesses, suggesting that part of the link between mental health and obesity can be related more to mediational or common factors than to obesity itself (Dickerson et al., 2006; de Las Cuevas et al., 2011). Social and lifestyle factors also play a role. A cross-sectional study found that the link between depression and obesity was partially mediated by lower physical and social activity in those with depression (de Wit et al., 2010). People with obesity are also highly stigmatized and the experience of social stigma can be related to increased depression in those with obesity (Myers and Rosen, 1999).

Medical studies and studies of animals indicate that effects of obesity on mental health may not be purely social but have 
a strong biological component. Obesity-linked, hypercaloric (in particular high-sugar, high refined fat) diets appear to create vulnerability to the development of mental disorder, such as anxiety, via their effects on neuroendocrine stress and the neural circuitry that supports the regulation of emotions (Baker et al., 2017; de Noronha et al., 2017). Obesity-prone rats (especially males) experience elevated anxiety upon gaining weight, suggesting a biological relationship between obesity and anxiety that extends beyond the social challenges that cloud the ability to determine direction of effects in humans (Alonso-Caraballo et al., 2019). In another study of mice fed a standard diet vs. a Western diet (defined as palatable, energydense food) for 20 weeks, mice fed a Western diet displayed impairments in spatial recognition memory and increased anxious behavior, as well as more susceptibility to depressive behavior after being immune suppressed (Andre et al., 2014). Mouse studies indicate that even exposure of a fetus to dietinduced maternal obesity can produce increased anxiety and impaired stress coping in offspring 3 months (young) and 12 months (aged) after birth, due to impairments in glucocorticoid receptors in the hypothalamus (Balsevich et al., 2016). The anxiety that comes with obesity appears to be reducible in animal models via measures to reduce chronic inflammation, such as dietary restriction and ibuprofen (Fourrier et al., 2019). Similarly, in humans, chronic inflammation, in the form of increased cytokines, such as tumor necrosis factor $\alpha$ and interleukin (IL)-1, often linked to diet and obesity, is associated with increased depression (Berk et al., 2013). Gastric bypass surgery for morbid obesity not only reduces obesity, but also improves mental health as well (Frezza et al., 2007). Further, decreasing Obesity Hypoventilation Syndrome in those with obesity via positive airway pressure therapy reduces symptoms of anxiety and depression (Argun Baris et al., 2016), again pointing to a reduction in obesity and obesity-related symptoms being related to a reduction in mental distress.

\section{Cross-Cultural Differences}

While meta-analyses have shown some fairly consistent results, much of the research has been conducted with Western North American and European populations. However, there appear to be some important, and underexplored cultural differences in the appearance of these effects in different countries and cultures. A comparison of Caucasians, Latinos, and African Americans indicates that later obesity is predicted similarly across groups by earlier anxiety and mood disorders, but that strength and significance are slightly stronger for Caucasians than for Latinos and African Americans (Bodenlos et al., 2011). In a study of children with obesity in Brazil, where there is a high prevalence of pediatric obesity (Aiello et al., 2015), children with obesity were not found to be more anxious than children in other weight categories (Araujo et al., 2017). Interestingly, there appear to be some class differences in the effects of obesity on mental health in certain countries, but not others. Obesity is concurrently linked to higher rates of several mental disorders in German women, including anxiety, regardless of socioeconomic status (Becker et al., 2001). However, a study in Mexico, a country with some of the highest rates of obesity in the world, found that while obesity was linked to depression in more affluent people, it was positively related to mental health in the poor. It was hypothesized that obesity is likely to be more accepted and less stigmatized among the poor in certain countries (Bargain and Zeidan, 2019), perhaps those where resources are seen as more scarce, or where obesity is more common. Finally, in a study of Chinese children, being an only child (which remains more common in China due to the now-expired one-child policy) is related to greater obesity. However, while obesity is related to more depression in sibling pairs, only children (i.e., singletons) with obesity reported lower depression than children with obesity in sibling pairs. Children without overweight did not differ in depression according to sibling relationship. There were no differences in anxiety among children with or without obesity or sibling pairs in this study (Peng et al., 2019). In sum, cultural differences exist in the relationship between obesity and mental health, with the relationship potentially reversed under certain socioeconomic and structural conditions.

\section{Obesity and Positive Mental Health Facets}

While historically, mental health has been defined de facto as the absence of psychopathology (Keyes, 2005; Prisciandaro and Roberts, 2009; Wittchen et al., 2011), this view is now recognized increasingly as overly simplistic, with positive mental health being a distinct and important construct for understanding the human condition (Prisciandaro and Roberts, 2009; Wittchen et al., 2011). A very rare few studies have found the potential for positive mental health or lower mental disorder accompanying obesity under certain circumstances, such as in the case of the poor in Mexico (Bargain and Zeidan, 2019), singletons in China (Peng et al., 2019), or no effect as in the case of the children in Brazil (Araujo et al., 2017). However, no known study has examined the relationship between obesity and positive aspects of mental health, such as sense of wellbeing, life satisfaction, or happiness. Traits, such as resilience and personal values, as well as states, such as current happiness and life satisfaction (Michael et al., 2007; Jacobi et al., 2014; Maercker et al., 2015; Lukat et al., 2016), are positively related to positive mental health (Haddadi and Besharat, 2010) and negatively associated with depressive symptoms (Brunwasser et al., 2009). It could be that an impact of obesity on resilience or happiness could mediate the relationship between obesity and mental distress or that the presence of positive traits or states could buffer those with obesity from mental distress. Further highlighting the importance of examining positive aspects of mental health separately from negative aspects, one recent large-scale longitudinal study of broad based mental disorders found that pathogenic predictors predict incidence and relapse of mental disorder diagnoses, while salutogenic factors predict remission over time (Lukat et al., 2016). It could be that obesity and positive mental health are related independently from mental distress and that the presence of salutogenic factors may serve as important buffers for those with overweight and obesity. 


\section{Present Study}

The present study is conducted within the "Bochum Optimism and Mental Health (BOOM) Project" (Margraf and Schneider, 2017), which aim to enhance integrated knowledge of the causes and consequences of positive mental health and mental health problems cross-culturally and over time. The present analysis, specifically, is a large-scale, cross-cultural, bi-national, and longitudinal investigation into the relationship between obesity and positive (life satisfaction, happiness, and resilience) and negative (anxiety and depression) mental health. Dimensional measures of mental health are examined, rather than categorical measures. Factors are examined across two time points (baseline and one-year follow-up), and across two countries (Germany and China). We limited the number of predictors for methodological reasons (Peduzzi et al., 1996). We hypothesized that obesity would generally be positively related to anxiety and depression, and negatively related to happiness over time. We expected health state, self-evaluation of attractiveness, and life satisfaction to partially but not fully mediate the links between obesity and mental state. We also explored resilience as a moderator of the pathways, and predicted a possible stronger effect of the moderator in China than in Germany, based on limited past research showing that the relationship between mental health and obesity can differ depending on family structure in China (Peng et al., 2019).

\section{MATERIALS AND METHODS}

\section{Procedure}

The present study utilizes a subset of data from the BOOM project, a large-scale, cross-cultural, and longitudinal investigation of risk and protective factors in mental health (Maercker et al., 2015; Margraf and Schneider, 2017; Margraf et al., 2020). For a comprehensive overview of the full study design, aims, measures, and participants, see Margraf and Schneider (2017). The Ethics Committee of the Faculty of Psychology of the Ruhr-University Bochum approved the study. Approval to administer the questionnaires was granted by the Faculty of Psychology at Ruhr-University Bochum on May 12, 2011 and renewed on September 2013. The approvals for the German site were communicated to the participating Chinese Universities who acknowledged and accepted these approvals.

\section{Participants Germany}

The German sample consisted of 364 (1,040 had data at baseline and 395 of them had data at follow-up) student participants with BMI at baseline greater than 18.5 recruited from Ruhr-University Bochum in 2013. Students were assessed via online survey. German students were recruited via an e-mailed invitation with a link leading to an online questionnaire. The link was sent to all students enrolled at Ruhr-University Bochum. Participants were offered an incentive to take part in a draw for a gift coupon or a tablet computer. Participants had the opportunity at the end of the surveys contact the respective coordinators of the surveys with any questions or concerns. No participants contacted the researchers with concerns about the topic of obesity or the connection between obesity and mental health or disorder.

\section{China}

As the data were anonymized from the very beginning of data collection, no statement by an institutional board/ethics committee was required to collect data in China. The final Chinese sample consisted of 9,007 (12,057 had data at both time points, but those who with no BMI-value or a BMI-value under 18.5 were excluded, leaving a total of 9,007) university students with BMI at baseline greater than 18.5 from the Capital Normal University Beijing, the Hebei United University, Shanghai Normal University, Guizhou Finance and Economics University, and Nanjing University. Participants, mainly freshmen, were recruited during their first study month via an invitation mail. The response rate was $94.5 \%$. Data were gathered via an online or a paper-pencil questionnaire administered in a group testing session. Participants received 10 RenMinBi (approximately 1.3 Euros) upon returning the questionnaire. As in Germany, participants in China had the opportunity at the end of the surveys contact the respective coordinators of the surveys with any questions or concerns. No participants contacted the researchers with concerns about the topic of obesity or the connection between obesity and mental health or disorder.

\section{Measures \\ Overview}

As far as possible, established brief standard instruments, such as the Depression Anxiety Stress Scales (e.g., DASS 21), were used to measure the constructs of interest. For all questionnaires used in the analysis, validated German versions exist. Chinese versions of the measures were developed when needed, by using the customary translation-back-translation method as recommended (Brislin, 1970). In cases of discrepancies, this procedure was repeated by the study team until complete agreement was achieved. Measures can be grouped according to the overall design of the research program. Table 1 provides an overview.

\section{General Outcomes Overweight and Obesity}

BMI was calculated based on self-reported weight and height, that is, the weight in kilograms divided by the square of the height in meters $\left(\mathrm{kg} / \mathrm{m}^{2}\right)$. Following recommendations of the (WHO, 2000), BMI was classified into four categories: $1=$ underweight $\quad\left(\mathrm{BMI}<18.5 \mathrm{~kg} / \mathrm{m}^{2}\right), \quad 2=$ normal $\quad$ weight $\left(18.5 \leq \mathrm{BMI}<25 \mathrm{~kg} / \mathrm{m}^{2}\right), \quad 3=$ overweight $\quad\left(25 \leq \mathrm{BMI}<30 \mathrm{~kg} / \mathrm{m}^{2}\right)$, and $4=$ obese $\left(\mathrm{BMI} \geq 30 \mathrm{~kg} / \mathrm{m}^{2}\right)$. Participants with underweight were not included in this study. The last two categories were merged into one "Overweight and Obesity" and coded as 1, while normal weight was coded as 0 . 
TABLE 1 | Descriptive statistics of sociodemographic variables and measures.

\begin{tabular}{|c|c|c|c|c|}
\hline & \multicolumn{2}{|c|}{ China $(n=9,007)$} & \multicolumn{2}{|c|}{ Germany ( $n=364)$} \\
\hline & Female $(n=5,182)$ & $\operatorname{Male}(n=3,825)$ & Female $(n=230)$ & Male $(n=134)$ \\
\hline \multicolumn{5}{|l|}{ Partnership BL } \\
\hline $\begin{array}{l}\text { Yes } \\
\text { No }\end{array}$ & $\begin{array}{c}732(14.15 \%) \\
4,441(85.85 \%)\end{array}$ & $\begin{array}{c}580(15.18 \%) \\
3,242(84.82 \%)\end{array}$ & $\begin{array}{c}123(53.48 \%) \\
107(46.52 \%)\end{array}$ & $\begin{array}{l}56(41.79 \%) \\
78(58.21 \%)\end{array}$ \\
\hline \multicolumn{5}{|c|}{ Overweight and Obesity BL } \\
\hline \multirow[t]{2}{*}{$\mathrm{No}$ (= normal weight) } & $\begin{array}{c}223(4.30 \%) \\
4,959(95.70 \%)\end{array}$ & $\begin{array}{c}460(12.03 \%) \\
3,365(87.97 \%)\end{array}$ & $\begin{array}{c}40(17.39 \%) \\
190(82.61 \%)\end{array}$ & $\begin{array}{c}25(18.66 \%) \\
109(81.34 \%)\end{array}$ \\
\hline & Mean(SD) & Mean(SD) & Mean(SD) & Mean(SD) \\
\hline Age_BL & $19.58(1.59)$ & $19.79(1.87)$ & $20.60(2.87)$ & $20.48(2.50)$ \\
\hline FASII_BL & $2.91(2.14)$ & $2.40(2.10)$ & $3.91(1.76)$ & $3.99(1.76)$ \\
\hline Resilience_BL & $58.44(8.03)$ & $59.14(8.89)$ & $58.67(9.33)$ & $60.14(8.05)$ \\
\hline Attractiveness_FU & $70.56(16.15)$ & $71.59(19.24)$ & $58.17(23.68)$ & $62.12(18.35)$ \\
\hline Health state_FU & 83.58 (13.09) & $83.11(14.37)$ & $72.92(24.17)$ & $75.78(21.26)$ \\
\hline Satisfaction with life_FU & $23.05(6.45)$ & $22.43(6.94)$ & $24.48(6.01)$ & $23.76(6.72)$ \\
\hline Subjective happiness_FU & $21.50(4.11)$ & $20.94(4.87)$ & $19.15(4.50)$ & $19.46(4.10)$ \\
\hline Anxiety_FU & $3.00(3.24)$ & 3.29 (3.94) & $3.40(3.86)$ & $2.70(2.94)$ \\
\hline Depression_FU & $2.25(3.04)$ & $2.99(3.98)$ & $4.60(4.70)$ & $4.22(4.00)$ \\
\hline
\end{tabular}

BL, baseline; FU, follow-up.

\section{Subjective Happiness}

Global subjective happiness was assessed using the four-item Subjective Happiness Scale (Lyubomirsky and Lepper, 1999). Participants responded on a 7-point Likert scale. The wording of anchor points depended on the question. Item scores are combined into a sum score with higher scores indicating higher happiness. Cronbach's alphas at baseline were 0.829 (Germany) and 0.776 (China).

\section{Depression, Anxiety, and Stress}

Anxiety and depression were assessed using the widely used Depression Anxiety Stress Scales (DASS-21; Henry and Crawford, 2005). This short form of the DASS-42 (Lovibond and Lovibond, 1995) assesses a broad range of psychological distress symptoms. It is composed of three 7-item subscales for depressive, anxiety, and stress symptoms over the past week. The subscales may serve as outcome measures and as screening and monitoring instruments (Ng et al., 2007; Bayram and Bilgel, 2008; Dahm et al., 2013). Items are rated on a 4-point Likert scale ranging from 0 (did not apply to me at all) to 3 (applied to me very much or most of the time). Responses can be averaged within subscale or across all three for a total item score. Psychometric properties are well established in both clinical and non-clinical samples (Henry and Crawford, 2005; Ng et al., 2007) and are comparable for the short and long versions (Lovibond and Lovibond, 1995; Antony et al., 1998). In addition, the present authors have unpublished data on file that show the scale to be appropriate for cross-cultural research, with measurement invariance across cultures. In the present study, Cronbach's alphas at baseline for depression were 0.886 (Germany) and 0.776 (China). Alphas for anxiety were 0.774 (Germany) and 0.737 (China).

\section{Mediators and Moderators} Resilience

Psychosocial stress resilience was assessed with an 11-item short version of the Wagnild and Young Resilience Scale (RS-14;
RS-11; Wagnild and Young, 1993). Participants responded to items, such as "I usually manage one way or another" on a scale ranging from 1 ("I disagree") to 7 ("I agree"). The RS-11 demonstrated good reliability and convergent validity in a German sample (Schumacher et al., 2005). Cronbach's alphas at baseline were 0.891 (Germany) and 0.793 (China).

\section{Attractiveness}

Attractiveness was assessed using a single item: "How good or bad do you think you look compared to other people's looks?" Participants rated themselves on a scale ranging from 0 ("completely ugly looking") to 100 ("completely beautiful looking”).

\section{Quality of Health}

Overall current quality of health was assessed using the EuroQol (EQ-VAS; EuroQol Group, 1990, 2013; Brooks, 1996). Participants rated current health status on a scale ranging from 0 (worst imaginable health) to 100 (best imaginable health). Validity of EQ-VAS is indicated by convergence with the five dimensional version of the EuroQol (EuroQol 5D), with WHO-5, and known clinical groups across several countries.

\section{Satisfaction With Life}

The satisfaction with life scale (Diener et al., 1985) consists of five items focusing on global life satisfaction. Ratings on a 7-point Likert scale ranging from 1 ("strongly disagree") to 7 ("strongly agree") indicate agreement with each item. Scores were averaged across items. Cronbach's alphas at follow-up were 0.881 (Germany) and 0.892 (China).

\section{Sociodemographic Predictors Basic Sociodemographic Predictors}

Sex, age, and relational status were assessed via self-report. 


\section{Family Affluence}

To ensure sufficient comparability across vastly different cultures, the Family Affluence Scale II (FAS II; Boyce et al., 2006) served as the main cross-cultural measure of socioeconomic circumstances. The FAS II is, a four-item measure of family wealth, developed in the WHO Health Behavior in School-aged Children Study. Questions include (either with 2 or 3 response alternatives): "Does your family own a car, van or truck?", "Do you have your own bedroom for yourself?", "During the past 12 months, how many times did you travel away on holiday with your family?", and "How many computers does your family own?". The FAS II total score is calculated by summing up the responses to these items. Convergent validity is established via correlations with the Gross National Product across 35 countries (Boyce et al., 2006). Cronbach's alphas at baseline were 0.315 (Germany) and 0.640 (China), consistent with past research (Schnohr et al., 2008), indicating that the FAS II has low internal consistency. But FAS II still has the potential to address many of the limitations of other proxy measures of socioeconomic status currently being used in research (Kehoe and O’Hare, 2010).

\section{STATISTICAL ANALYSES}

All analyses are calculated with SPSS 24 (Corp, 2016) and PROCESS V3.4 (Hayes, 2013). A series of models are conducted for each country and gender separately to specify the relations between variables in this study. The program PROCESS was used to conduct our mediator-moderator analyses, and PROCESS does not produce measures of fit for the entire model (Hayes et al., 2017). Therefore, we did not report the model fit indices. Obesity and overweight at baseline are used as a predictor of happiness, depression, and anxiety at follow-up, while using attractiveness, health, and satisfaction with life at follow-up as mediators and resilience at baseline as moderator. Age, FAS II, and having a partner at baseline are all controlled in the models. Tests of moderation within path models are conducted. The bootstrapping method with bias corrected confidence interval (MacKinnon et al., 2004) is estimated to test the significance of mediators. In this study, the $95 \%$ confidence interval of the indirect effects is calculated with 5,000 bootstrap resamplings (Preacher and Hayes, 2008). As having obesity/overweight at baseline is coded as a dichotomous variable, no simple slope analyses are conducted for any significant interaction paths.

Missing values were generally between 0.0 and 3.3\%, depending on the measure. Participants with missing data were deleted pairwise from the analyses (meaning they were deleted from analyses in which those questionnaires were used). Internal consistency is computed with Cronbach's $\alpha$ coefficient. Cronbach's $\alpha>0.70$ indicates acceptable, $>0.80$ good, and $>0.90$ excellent internal consistency (Kline, 2000).

\section{RESULTS}

\section{Descriptive Statistics}

Table 1 presents data on participant demographics and descriptive statistics for the predictors and outcomes at baseline.
Approximately $49 \%$ of Germans were in a partnership, while only $14.6 \%$ of Chinese participants were in a partnership. About $18 \%$ of Germans in the current study had overweight or obesity, while $7.6 \%$ of Chinese participants had the same. The German participants rated their family affluence as slightly higher, their attractiveness as lower, health state lower, and depression higher than the Chinese participants. Between country differences were not evaluated for significance. Chinese participants had a mean age of $19.67(S D=1.72)$, and German participants had a mean age of $20.55(S D=2.74)$.

\section{Correlations}

The correlations among the psychological predictors are shown in Table 2A (Germany) and Table 2B (China). Pearson's zeroorder correlations indicated that overweight/obesity was significantly related to lower family affluence attractiveness, health, and life satisfaction, but not depression or anxiety in German female participants. Overweight/obesity was only significantly related to higher family affluence in German male participants. It was related to slightly higher family affluence, lower resilience, and lower attractiveness, but not to life satisfaction, depression, or anxiety in Chinese females. Overweight/obesity was, however, related to higher family affluence, later depression, and anxiety in Chinese males.

\section{Structural Equation Model}

The general model is presented in Figure 1. The model was tested for three different outcomes happiness, anxiety, and depression, and was tested separately for the German and Chinese samples. Table 3 indicates the effect strength and significance for each pathway with happiness as the outcome. Table 4 indicates the same model results, except with depression as the outcome. Table 5 indicates the model results with anxiety as the outcome.

Results indicated that in Chinese male students, attractiveness t 2 and health state $\mathrm{t} 2$ are significant mediators for depression t2 and happiness t2. Further, health state t2 is a significant mediator for anxiety $\mathrm{t} 2$. In Chinese female students, attractiveness $\mathrm{t} 2$ is a significant mediator for depression $\mathrm{t} 2$, anxiety $\mathrm{t} 2$, and happiness t2. Further, resilience t1 is a significant moderator only for happiness $\mathrm{t} 2$. For Chinese females with low resilience at baseline, overweight/obesity is related to higher happiness at the follow-up than normal weight is. However, for Chinese females with high resilience at baseline, overweight/obesity leads to lower subjective happiness at the follow-up than normal weight. In German male students, there was no significant mediator or moderator. In German female students, satisfaction with life $\mathrm{t} 2$ is a significant mediator for depression $\mathrm{t} 2$ and anxiety $\mathrm{t} 2$. Further, attractiveness $\mathrm{t} 2$ and satisfaction with life $\mathrm{t} 2$ are significant mediators for happiness $\mathrm{t} 2$.

\section{DISCUSSION}

To our knowledge, this is the first longitudinal prospective study to examine the relationship between obesity and positive 
TABLE 2A | Bivariate correlations among the psychological predictors within Germany, with female below diagonal and male above diagonal.

\begin{tabular}{|c|c|c|c|c|c|c|c|c|c|c|c|}
\hline & $\begin{array}{c}\text { Partnership } \\
\text { BL }\end{array}$ & $\begin{array}{c}\text { Overweight } \\
\text { and Obesity } \\
\text { BL }\end{array}$ & Age BL & FASII BL & $\begin{array}{c}\text { Resilience } \\
\text { BL }\end{array}$ & $\begin{array}{c}\text { Attractiveness } \\
\text { FU }\end{array}$ & $\begin{array}{l}\text { Health } \\
\text { state FU }\end{array}$ & $\begin{array}{l}\text { Satisfaction } \\
\text { with life FU }\end{array}$ & $\begin{array}{c}\text { Subjective } \\
\text { happiness FU }\end{array}$ & $\begin{array}{l}\text { Anxiety } \\
\text { FU }\end{array}$ & $\begin{array}{c}\text { Depression } \\
\text { FU }\end{array}$ \\
\hline Partnership BL & 1 & -0.056 & 0.123 & -0.044 & -0.015 & -0.038 & -0.025 & 0.166 & $0.198^{*}$ & -0.043 & -0.058 \\
\hline OverweightandObesity BL & -0.124 & 1 & 0.169 & $0.178^{*}$ & -0.035 & -0.118 & 0.006 & 0.026 & 0.036 & 0.141 & 0.143 \\
\hline Age_BL & 0.084 & $0.177^{* *}$ & 1 & $-0.175^{*}$ & -0.138 & -0.12 & $-0.199^{*}$ & $-0.216^{*}$ & -0.087 & 0.156 & $0.301^{* *}$ \\
\hline FASII_BL & 0.041 & $-0.140^{*}$ & $-0.151^{*}$ & 1 & 0.08 & 0.134 & 0.11 & $0.203^{*}$ & 0.146 & -0.042 & -0.107 \\
\hline Resilience_BL & 0.118 & -0.093 & 0.071 & 0.126 & 1 & $0.206^{*}$ & $0.255^{* *}$ & $0.434^{* * *}$ & $0.411^{* * *}$ & $-0.365^{* *}$ & $-0.394^{* *}$ \\
\hline Attractiveness_FU & 0.092 & $-0.195^{* *}$ & -0.109 & 0.043 & $0.163^{*}$ & 1 & $0.283^{* *}$ & 0.152 & $0.289^{* *}$ & -0.142 & $-0.231^{* *}$ \\
\hline Health state_FU & 0.099 & $-0.142^{*}$ & -0.114 & $0.139^{*}$ & 0.066 & $0.367^{* *}$ & 1 & $0.342^{* *}$ & $0.284^{* * *}$ & $-0.341^{* *}$ & $-0.351^{* *}$ \\
\hline Satisfaction with life_FU & $0.163^{*}$ & $-0.219^{* *}$ & -0.082 & $0.286^{* *}$ & $0.416^{* *}$ & $0.393^{* *}$ & $0.279^{* *}$ & 1 & $0.522^{* *}$ & $-0.282^{* *}$ & $-0.386^{* *}$ \\
\hline Subjective happiness_FU & $0.173^{* *}$ & -0.11 & 0.043 & $0.131^{* *}$ & $0.455^{* *}$ & $0.373^{* *}$ & $0.178^{* *}$ & $0.699^{* *}$ & 1 & $-0.294^{* * *}$ & $-0.489^{* *}$ \\
\hline Anxiety_FU & -0.109 & 0.079 & 0 & $-0.208^{*}$ & $-0.138^{*}$ & $-0.147^{*}$ & $-0.198^{* *}$ & $-0.392^{* *}$ & $-0.381^{* *}$ & 1 & $0.665^{* *}$ \\
\hline Depression_FU & $-0.148^{*}$ & 0.109 & 0.02 & $-0.174^{* *}$ & $-0.272^{* *}$ & $-0.350^{* * *}$ & $-0.259^{* *}$ & $-0.624^{* *}$ & $-0.555^{* *}$ & $0.670^{*}$ & 1 \\
\hline
\end{tabular}

" $p<0.05$, two tailed; " $p<0.01$, two tailed. $B L=$ baseline, $F U=$ follow-up

TABLE 2B | Bivariate correlations among the psychological predictors within China, with female below diagonal and male above diagonal.

\begin{tabular}{|c|c|c|c|c|c|c|c|c|c|c|c|}
\hline & $\begin{array}{c}\text { Partnership } \\
\text { BL }\end{array}$ & $\begin{array}{c}\text { Overweight } \\
\text { and Obesity } \\
\text { BL }\end{array}$ & Age BL & FASII BL & $\begin{array}{c}\text { Resilience } \\
\text { BL }\end{array}$ & $\begin{array}{c}\text { Attractiveness } \\
\text { FU }\end{array}$ & $\begin{array}{l}\text { Health } \\
\text { state FU }\end{array}$ & $\begin{array}{l}\text { Satisfaction } \\
\text { with life FU }\end{array}$ & $\begin{array}{c}\text { Subjective } \\
\text { happiness FU }\end{array}$ & $\begin{array}{l}\text { Anxiety } \\
\text { FU }\end{array}$ & $\begin{array}{c}\text { Depression } \\
\text { FU }\end{array}$ \\
\hline Partnership BL & 1 & 0.003 & $0.157^{* *}$ & $0.106^{* *}$ & 0.016 & $0.056^{* *}$ & $0.033^{*}$ & $0.070^{* *}$ & $0.051^{* *}$ & -0.019 & -0.014 \\
\hline OverweightandObesity BL & $-0.037^{* *}$ & 1 & 0.006 & $0.121^{* *}$ & -0.024 & $-0.087^{* *}$ & $-0.061^{* *}$ & 0.03 & -0.011 & $0.058^{* *}$ & $0.055^{* *}$ \\
\hline Age_BL & $0.195^{* *}$ & -0.021 & 1 & $-0.154^{* *}$ & $0.037^{*}$ & $0.055^{* *}$ & $0.069^{* *}$ & 0.022 & 0.022 & -0.025 & -0.022 \\
\hline FASII_BL & $0.055^{* *}$ & $0.037^{* *}$ & $-0.148^{* *}$ & 1 & $0.046^{* *}$ & $-0.051^{* *}$ & $-0.094^{* *}$ & $0.094^{* *}$ & 0.018 & 0.009 & 0.012 \\
\hline Resilience_BL & $0.034^{*}$ & $-0.034^{*}$ & $0.034^{* *}$ & $0.070^{* * *}$ & 1 & $0.137^{* *}$ & $0.139^{* * *}$ & $0.216^{* *}$ & $0.252^{* *}$ & $-0.192^{* *}$ & $-0.204^{* *}$ \\
\hline Attractiveness_FU & $0.093^{* *}$ & $-0.031^{*}$ & $0.052^{* *}$ & $0.052^{* * *}$ & $0.163^{* *}$ & 1 & $0.356^{* * *}$ & $0.166^{* *}$ & $0.257^{* *}$ & $-0.159^{* * *}$ & $-0.176^{* *}$ \\
\hline Health state_FU & 0.013 & -0.019 & 0.006 & $-0.080^{* *}$ & $0.154^{* *}$ & $0.270^{* *}$ & 1 & $0.232^{* *}$ & $0.319^{* *}$ & $-0.255^{* *}$ & $-0.260^{* *}$ \\
\hline Satisfaction with life_FU & $0.086^{* * *}$ & 0.009 & $0.044^{*}$ & $0.140^{* *}$ & $0.194^{* * *}$ & $0.215^{* *}$ & $0.204^{* * *}$ & 1 & $0.542^{* *}$ & $-0.227^{* *}$ & $-0.237^{* *}$ \\
\hline Subjective happiness_FU & $0.064^{* *}$ & -0.006 & $0.048^{* *}$ & $0.054^{* *}$ & $0.262^{* *}$ & $0.255^{* *}$ & $0.322^{* *}$ & $0.492^{* *}$ & 1 & $-0.339^{* *}$ & $-0.371^{* *}$ \\
\hline Anxiety_FU & -0.027 & 0 & $-0.064^{* *}$ & $-0.044^{* *}$ & $-0.184^{* *}$ & $-0.156^{* *}$ & $-0.286^{* *}$ & $-0.237^{* * *}$ & $-0.320^{* *}$ & 1 & $0.881^{* * *}$ \\
\hline Depression_FU & $-0.038^{* * *}$ & 0.008 & $-0.043^{* *}$ & $-0.039^{* *}$ & $-0.210^{* * *}$ & $-0.167^{* *}$ & $-0.286^{* *}$ & $-0.262^{* *}$ & $-0.364^{* *}$ & $0.789^{* *}$ & 1 \\
\hline
\end{tabular}

" $p<0.05$, two tailed; " $p<0.01$, two tailed. $B L=$ baseline, $F U=$ follow-up. 


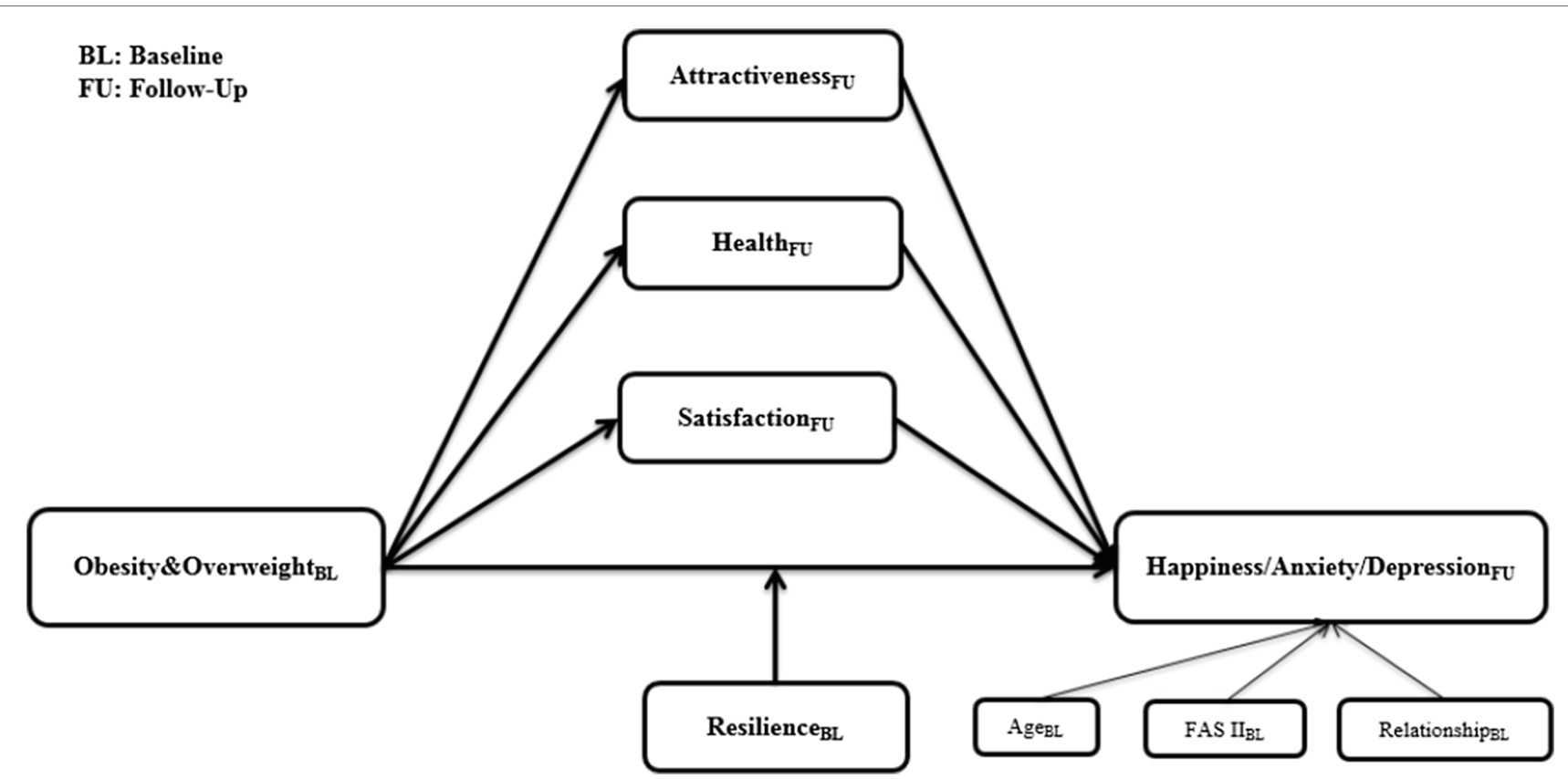

FIGURE 1 | Model with attractiveness, health state, and satisfaction from follow-up as mediators and resilience from baseline as moderator.

TABLE 3 | Summary of mediated and moderated regression analyses predicting happiness.

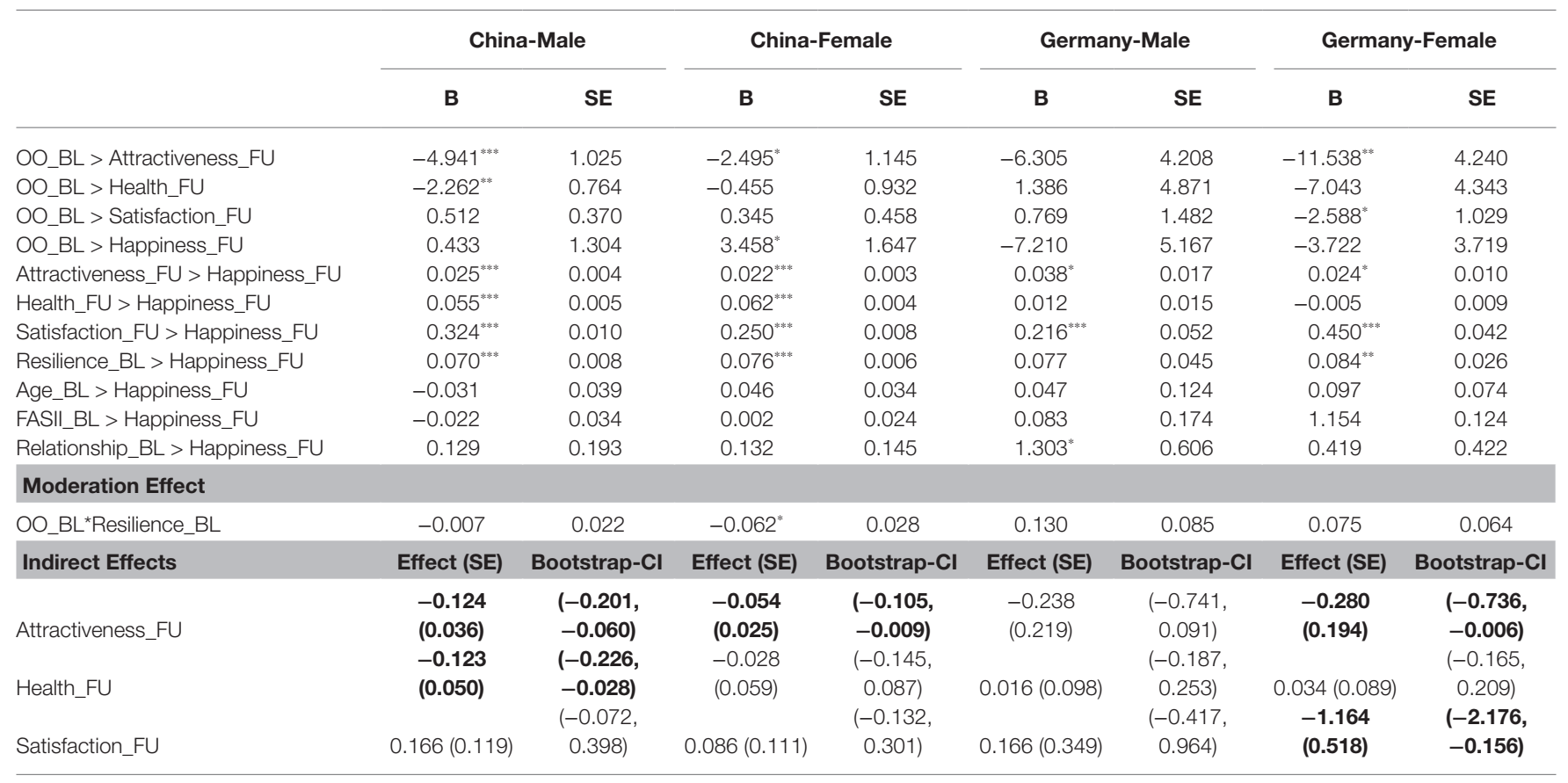

Significant effects in bold; B, unstandardized coefficient; and SE, standard error. " $p<0.05 ; " * * 0.01 ;{ }^{* * *} p<0.001$. BL = baseline, FU = follow-up.

and mental health and obesity in a cross-national sample. Results from zero-order correlations indicated that overweight/ obesity at baseline was related to lower concurrent family affluence and lower follow-up attractiveness, health, and life satisfaction, but not related to follow-up depression or anxiety in German female participants. Overweight/obesity was only significantly related to higher family affluence in German male participants. It was related to slightly higher family affluence, lower resilience, and lower attractiveness, but not to life satisfaction, depression, or anxiety in Chinese females. It was, however, related to increased family affluence, anxiety, and depression in Chinese males. The lack of associations between obesity and mental health in German men and women and Chinese women is in contrast to prior research. Indeed, several 


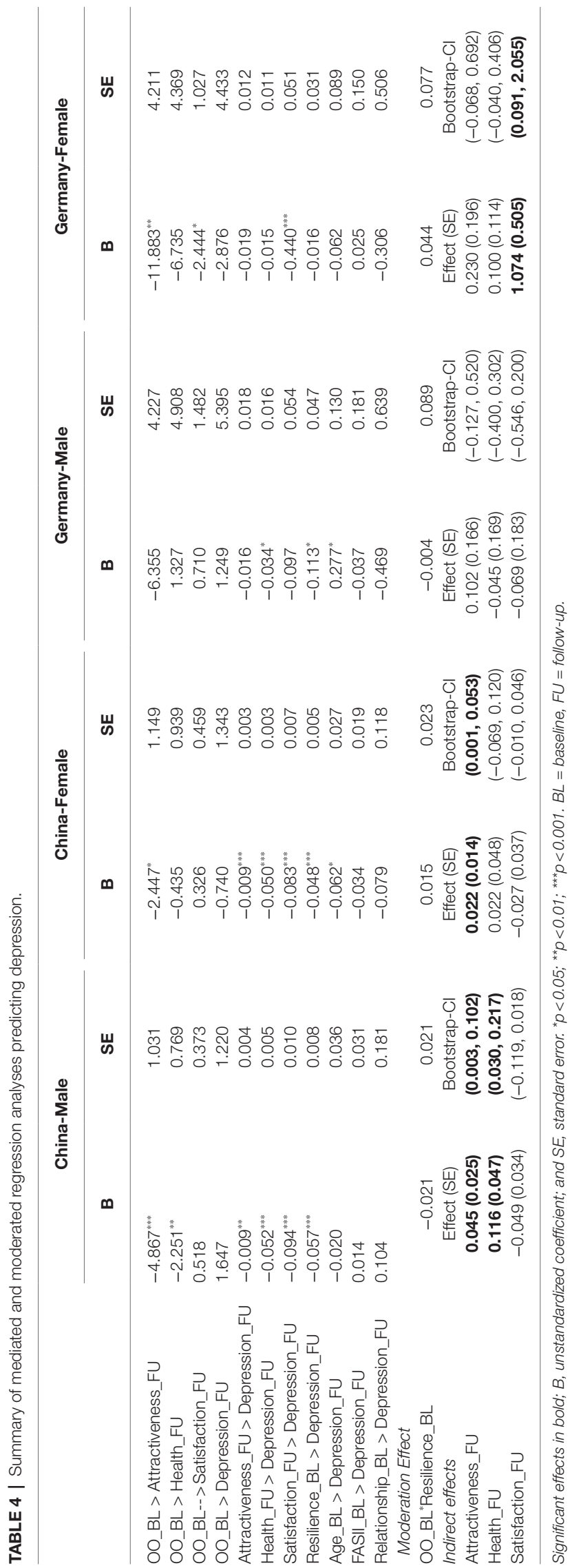

large-scale studies and meta-analyses have documented the relationship between overweight and increased anxiety and depression with moderate effects (OR in the range of 1.2-1.5) across 13 different countries in the World Mental Health Surveys (Scott et al., 2008), and between overweight and anxiety across 25 studies included in a large meta-analysis, with effects similar across sex (Amiri and Behnezhad, 2019), and across 16 studies in Gariepy et al. (2010). In a community study of over 36,000 Canadians, lifetime major depression was related to concurrent obesity with an odds ratio of 1.46 in females, though not related to obesity in males in this study (McIntyre et al., 2006). These findings make our own results even more perplexing, as we found a link between obesity and anxiety/depression in Chinese men, but not women. It could be that the sex difference result is dependent on culture, and we have no comprehensive study of Chinese sex differences until now. There are certainly cultural differences in these effects. A meta-analysis of the longitudinal bidirectional relationship between obesity/overweight and depression across 15 studies demonstrated that prediction of depression baseline obesity was stronger for Americans than Europeans. However, moderators of these effects across studies in the meta-analysis, including sex and age, were not significant (Luppino et al., 2010). Then again, some individual studies do find a sex effect, but the direction can be contradictory. Anxiety was a particular risk factor for later obesity in adolescent males in one study of United States teens (Roberts and Duong, 2016). Yet, another study found effects for United States female adolescents, but not for males (Anderson et al., 2007). Gender effects may be unstable, dependent on culture, or only apparent in teens, and perhaps college students, as we found in the present study.

Though zero-order correlations indicated that the expected obesity and mental health effects were only present in Chinese males, mediational analysis guidelines suggest that significant total effects (i.e., zero-order correlations) are not a necessary condition for examining mediational or indirect effects (Rucker et al., 2011). Thus, we did proceed to examine the relationships between obesity and outcomes in the planned structural equation models. The structural equation models indicated that in Chinese male students, attractiveness $\mathrm{t} 2$ and health state $\mathrm{t} 2$ were significant mediators for depression $\mathrm{t} 2$ and happiness t2. Further, health state t2 was a significant mediator for anxiety $\mathrm{t} 2$. That is, the relationship between obesity and mental health in Chinese males mediated by the time two assessments of one's own attractiveness and health.

In Chinese female students, attractiveness $\mathrm{t} 2$ was a significant mediator for depression $\mathrm{t} 2$, anxiety $\mathrm{t} 2$, and happiness $\mathrm{t} 2$. That is, in Chinese females, the relationship between obesity and mental health was significantly mediated by later attractiveness. Further, resilience $\mathrm{t} 1$ is a significant moderator, but only for happiness t2. That is, for Chinese females with low resilience at baseline, overweight/obesity is related to higher happiness at the follow-up than normal weight is. However, for Chinese females with high resilience at baseline, overweight/obesity leads to lower subjective happiness at the follow-up than normal weight. 
TABLE 5 | Summary of mediated and moderated regression analyses predicting anxiety.

\begin{tabular}{|c|c|c|c|c|c|c|c|c|}
\hline & \multicolumn{2}{|c|}{ China-Male } & \multicolumn{2}{|c|}{ China-Female } & \multicolumn{2}{|c|}{ Germany-Male } & \multicolumn{2}{|c|}{ Germany-Female } \\
\hline & B & SE & B & SE & B & SE & B & SE \\
\hline OO_BL > Attractiveness_FU & $-4.730^{* * * *}$ & 1.028 & $-2.548^{*}$ & 1.154 & -6.305 & 4.208 & $-12.208^{* * *}$ & 4.185 \\
\hline OO_BL $>$ Health_FU & $-2.167^{* *}$ & 0.765 & -0.466 & 0.942 & 1.386 & 4.871 & -7.734 & 4.360 \\
\hline OO_BL > Satisfaction_FU & 0.506 & 0.372 & -0.291 & 0.461 & 0.769 & 1.482 & $-2.484^{*}$ & 1.028 \\
\hline OO_BL-->Anxiety_FU & 1.326 & 1.210 & -0.770 & 1.473 & 5.832 & 4.141 & -0.114 & 4.379 \\
\hline Attractiveness_FU $>$ Anxiety_FU & -0.006 & 0.004 & $-0.010^{* * * *}$ & 0.003 & 0.004 & 0.014 & 0.005 & 0.012 \\
\hline Health_FU > Anxiety_FU & $-0.053^{* * * *}$ & 0.005 & $-0.055^{* * *}$ & 0.003 & $-0.034^{* *}$ & 0.012 & -0.012 & 0.011 \\
\hline Satisfaction_FU $>$ Anxiety_FU & $-0.089^{* * *}$ & 0.010 & $-0.076^{* * *}$ & 0.007 & -0.032 & 0.042 & $-0.236^{* * *}$ & 0.053 \\
\hline Resilience_BL > Anxiety_FU & $-0.051^{* * * *}$ & 0.008 & $-0.044^{* * * *}$ & 0.006 & $-0.078^{*}$ & 0.036 & 0.012 & 0.030 \\
\hline Age_BL $>$ Anxiety_FU & -0.025 & 0.036 & $-0.126^{* * *}$ & 0.030 & 0.048 & 0.100 & -0.069 & 0.087 \\
\hline FASII_BL > Anxiety_FU & 0.009 & 0.031 & $-0.055^{* *}$ & 0.021 & -0.008 & 0.139 & -0.233 & 0.150 \\
\hline Relationship_BL > Anxiety_FU & 0.062 & 0.179 & 0.080 & 0.128 & -0.254 & 0.485 & -0.119 & 0.510 \\
\hline \multicolumn{9}{|l|}{ Moderation Effect } \\
\hline OO_BL*Resilience_BL & -0.014 & 0.020 & 0.011 & 0.025 & -0.081 & 0.068 & 0.002 & 0.076 \\
\hline Indirect Effects & Effect (SE) & Bootstrap-CI & Effect (SE) & Bootstrap-CI & Effect (SE) & Bootstrap-Cl & Effect (SE) & Bootstrap-Cl \\
\hline Attractiveness_FU & $0.026(0.022)$ & $\begin{array}{c}(-0.012, \\
0.072)\end{array}$ & $0.025(0.014)$ & $\begin{array}{c}(\mathbf{0 . 0 1 4}, \mathbf{0 . 0 0 3}) \\
(-0.079\end{array}$ & $\begin{array}{c}-0.027 \\
(0.103) \\
-0.046\end{array}$ & $\begin{array}{c}(-0.240 \\
0.193) \\
(-0.345\end{array}$ & $\begin{array}{l}-0.059 \\
(0.188)\end{array}$ & $\begin{array}{c}(-0.471 \\
0.306) \\
(-0.062\end{array}$ \\
\hline Health_FU & $0.115(0.049)$ & $(0.025,0.216)$ & $0.026(0.054)$ & $0.134)$ & $(0.162)$ & $0.316)$ & $0.092(0.121)$ & $0.418)$ \\
\hline Satisfaction_FU & $\begin{array}{l}-0.045 \\
(0.033)\end{array}$ & $\begin{array}{c}(-0.112 \\
0.017)\end{array}$ & $\begin{array}{l}-0.022 \\
(0.035)\end{array}$ & $\begin{array}{c}(-0.089 \\
0.047)\end{array}$ & $\begin{array}{l}-0.024 \\
(0.092)\end{array}$ & $\begin{array}{c}(-0.276 \\
0.099)\end{array}$ & $0.587(0.328)$ & $(0.047,1.324)$ \\
\hline
\end{tabular}

Significant effects in bold; B, unstandardized coefficient; and SE, standard error. ${ }^{*} p<0.05 ;{ }^{* *} p<0.01 ;{ }^{* * *} p<0.001$. BL $=$ baseline, FU = follow-up.

In German male students, there was no significant mediator or moderator. In German female students, satisfaction with life t2 is a significant mediator for depression $\mathrm{t} 2$ and anxiety $\mathrm{t} 2$. Further, attractiveness $\mathrm{t} 2$ and satisfaction with life $\mathrm{t} 2$ are significant mediators for happiness $\mathrm{t} 2$. That is, although there was not a direct link between obesity and mental health (anxiety and depression) in German females, the structural equation model pointed to some significant mediational effects flowing through life satisfaction (on depression, anxiety, and happiness) and attractiveness (on happiness). In sum, obesity was not related to later mental health in the zero-order correlations for any group except for Chinese males. In Chinese males, obesity was significantly correlated with later depression and anxiety. Obesity was related to later attractiveness (Chinese and German females, and Chinese males), health (German females and Chinese males), and life satisfaction (German females), which were, in turn, related to time two mental health. Further, for Chinese females with low resilience, obesity can correlate with higher later happiness, whereas for Chinese females with high resilience, obesity is related to lower later happiness.

While not all who are overweight experience mental distress, for those for whom obesity is related to mental distress, it may be the case that reductions in obesity could foster some improvements in mental health. Some research indicates that weight loss is associated with improvements in mental health functioning (Carmichael et al., 2001; Jarvholm et al., 2012), suggesting that a reduction in obesity status leads to a reduction in mental distress. However, other health scientists have found that a focus on weight reduction in interventions can be counterproductive in improving both physical and mental wellbeing and that a focus on improving health rather than reducing weight leads to better outcomes (Bacon, 2010).
The present study has a number of strengths, including the large sample size, its thorough assessments using standardized instruments and follow-up interview over a period of 17 months. Because of the homogenous sample, age, gender, and socioeconomic characteristics were less likely to confound the effects of the psychological predictors. Moreover, we investigated the psychological predictors within a longitudinal design. Although there are several strengths associated with the study, there are also limitations. First, it is not possible to draw conclusions about causation, as this study did not employ an experimental design. Second, the present study examined effects in college students, and results may not be generalizable to populations of older or other non-college-attending adults. Third, obesity and overweight were coded as binary variables, when in reality weight exists on a linear scale. Fourth, the present study used self-report data. It may be that clinical reports of obesity and mental health would provide added reliability to the data. Fifth, there were fewer German than Chinese participants, and it may be that with more German participants, we would have had increased power to observe small effects. Sixth, this study used self-report measures, which may be subject to bias and are not as objective as multi-rater or observational methods. Seventh, participants were given an incentive to participate, which may have influenced the sample. Finally, while all ethical guidelines were followed, we agree with a limitation of the study pointed out by an anonymous reviewer: That is, participants filling out self-report questionnaires on mental health should be provided with a follow-up resource for mental health concerns.

Overweight/obesity is related to mental health across many studies and meta-analyses. However, in this study, 
total effects between overweight/obesity and follow-up mental health were only found significant in Chinese males. The effects between overweight/obesity and follow-up mental health were significantly mediated by follow-up attractiveness, or health state, or satisfaction in German females, Chinese females, and Chinese male students, but no significant indirect effects were found in German male students. This highlights the possible importance of culture in examining these effects. These results await replication and should be taken in the context of the broad literature on this topic. They may support newer foci on health and self-image rather than weight in treating mental health in obesity. The relationship between obesity and mental health in prior research still points to need for a multi-tiered public health approach to addressing mental health in those with obesity, including community intervention and prevention, as well as individual support (Bazyk and Winne, 2013).

\section{DATA AVAILABILITY STATEMENT}

The raw data supporting the conclusions of this article will be made available by the authors, without undue reservation.

\section{REFERENCES}

Aiello, A. M., Marques de Mello, L., Souza Nunes, M., Soares da Silva, A., and Nunes, A. (2015). Prevalence of obesity in children and adolescents in Brazil: A meta-analysis of cross-sectional studies. Curr. Pediatr. Rev. 11, 36-42. doi: 10.2174/1573396311666150501003250

Albohn-Kuhne, C., and Rief, W. (2011). Shame, guilt and social anxiety in obesity with binge-eating disorder. Psychother. Psychosom. Med. Psychol. 61, 412-417. doi: 10.1055/s-0031-1284334

Allison, D. B., Newcomer, J. W., Dunn, A. L., Blumenthal, J. A., Fabricatore, A. N., Daumit, G. L., et al. (2009). Obesity among those with mental disorders: a National Institute of Mental Health meeting report. Am. J. Prev. Med. 36, 341-350. doi: 10.1016/j.amepre.2008.11.020

Alonso-Caraballo, Y., Hodgson, K. J., Morgan, S. A., Ferrario, C. R., and Vollbrecht, P. J. (2019). Enhanced anxiety-like behavior emerges with weight gain in male and female obesity-susceptible rats. Behav. Brain Res. 360, 81-93. doi: $10.1016 /$ j.bbr.2018.12.002

Amiri, S., and Behnezhad, S. (2019). Obesity and anxiety symptoms: a systematic review and meta-analysis. Neuropsychiatrie 33, 72-89. doi: 10.1007/s40211-019-0302-9

Anderson, S. E., Cohen, P., Naumova, E. N., Jacques, P. F., and Must, A. (2007). Adolescent obesity and risk for subsequent major depressive disorder and anxiety disorder: prospective evidence. Psychosom. Med. 69, 740-747. doi: 10.1097/PSY.0b013e31815580b4

Andre, C., Dinel, A. L., Ferreira, G., Laye, S., and Castanon, N. (2014). Dietinduced obesity progressively alters cognition, anxiety-like behavior and lipopolysaccharide-induced depressive-like behavior: focus on brain indoleamine 2,3-dioxygenase activation. Brain Behav. Immun. 41, 10-21. doi: 10.1016/j.bbi.2014.03.012

Antony, M. M., Bieling, P. J., Cox, B. J., Enns, M. W., and Swinson, R. P. (1998). Psychometric properties of the 42-item and 21-item versions of the depression anxiety stress scales in clinical groups and a community sample. Psychol. Assess. 10, 176-181. doi: 10.1037/1040-3590.10.2.176

Araujo, D. S., Marquezin, M., Barbosa, T. S., Fonseca, F., Fegadolli, C., and Castelo, P. M. (2017). Assessment of quality of life, anxiety, socio-economic factors and caries experience in Brazilian children with overweight and obesity. Int. J. Dent. Hyg. 15, e156-e162. doi: 10.1111/idh.12248

Argun Baris, S., Tuncel, D., Ozerdem, C., Kutlu, H., Onyilmaz, T., Basyigit, I., et al. (2016). The effect of positive airway pressure therapy on neurocognitive

\section{ETHICS STATEMENT}

The studies involving human participants were reviewed and approved by the Ruhr-University Bochum. Written informed consent from the participants' legal guardians/next of kin was not required to participate in this study in accordance with the national legislation and the institutional requirements.

\section{AUTHOR CONTRIBUTIONS}

KL wrote the first draft and revised the manuscript. XZ analyzed the data, designed the analyses, and wrote the results. SS and JM designed the study, managed the study, planned the analyses, and provided the funding. All authors contributed to the article and approved the submitted version.

\section{FUNDING}

This study was supported by the Alexander von Humboldt Professorship awarded to Jürgen Margraf by the Alexander von Humboldt Foundation.

functions, depression and anxiety in obesity hypoventilation syndrome. Multidiscip. Respir. Med. 11:35. doi: 10.1186/s40248-016-0071-2

Bacon, L. (2010). Health at every Size: The Surprising Truth about your Weight. Dallas, TX: BenBella Books.

Baker, K. D., Loughman, A., Spencer, S. J., and Reichelt, A. C. (2017). The impact of obesity and hypercaloric diet consumption on anxiety and emotional behavior across the lifespan. Neurosci. Biobehav. Rev. 83, 173-182. doi: 10.1016/j.neubiorev.2017.10.014

Balsevich, G., Baumann, V., Uribe, A., Chen, A., and Schmidt, M. V. (2016). Prenatal exposure to maternal obesity alters anxiety and stress coping Behaviors in aged mice. Neuroendocrinology 103, 354-368. doi: $10.1159 / 000439087$

Bargain, O., and Zeidan, J. (2019). Heterogeneous effects of obesity on mental health: evidence from Mexico. Health Econ. 28, 447-460. doi: 10.1002/ hec. 3852

Bayram, N., and Bilgel, N. (2008). The prevalence and socio-demographic correlations of depression, anxiety and stress among a group of university students. Soc. Psychiatry Psychiatr. Epidemiol. 43, 667-672. doi: 10.1007/ s00127-008-0345-x

Bazyk, S., and Winne, R. (2013). A multi-tiered approach to addressing the mental health issues surrounding obesity in children and youth. Occup. Ther. Health Care 27, 84-98. doi: 10.3109/07380577.2013.785643

Becker, E. S., Margraf, J., Turke, V., Soeder, U., and Neumer, S. (2001). Obesity and mental illness in a representative sample of young women. Int. J. Obes. Relat. Metab. Disord. 25 Suppl_1, S5-S9. doi:10.1038/sj.ijo.0801688

Berk, M., Williams, L. J., Jacka, F. N., O'Neil, A., Pasco, J. A., Moylan, S., et al. (2013). So depression is an inflammatory disease, but where does the inflammation come from? BMC Med. 11:200. doi: 10.1186/1741-7015-11-200

Bodenlos, J. S., Lemon, S. C., Schneider, K. L., August, M. A., and Pagoto, S. L. (2011). Associations of mood and anxiety disorders with obesity: comparisons by ethnicity. J. Psychosom. Res. 71, 319-324. doi: 10.1016/j. jpsychores.2011.03.004

Boyce, W., Torsheim, T., Currie, C., and Zambon, A. (2006). The family affluence scale as a measure of national wealth: validation of an adolescent self-report measure. Soc. Indic. Res. 78, 473-487. doi: 10.1007/s11205-005-1607-6

Bradshaw, T., and Mairs, H. (2014). Obesity and serious mental ill health: A critical review of the literature. Healthcare 2, 166-182. doi: 10.3390/ healthcare2020166 
Brislin, R. W. (1970). Back-translation for cross-cultural research. J. Cross-Cult. Psychol. 1, 185-216. doi: 10.1177/135910457000100301

Brooks, R. (1996). EuroQol: The current state of play. Health Policy 37, 53-72. Brumpton, B., Langhammer, A., Romundstad, P., Chen, Y., and Mai, X. M. (2013). The associations of anxiety and depression symptoms with weight change and incident obesity: The HUNT study. Int. J. Obes. 37, 1268-1274. doi: $10.1038 /$ ijo.2012.204

Brunwasser, S. M., Gillham, J. E., and Eric, S. K. (2009). A meta-analytic review of the Penn Resiliency Program's effect on depressive symptoms. J. Consult. Clin. Psychol. 11, 708-715. doi: 10.1037/a0017671

Calderon, C., Forns, M., and Varea, V. (2009). Adolescent obesity: anxiety, cognitive and behavioural symptoms characteristic of eating disorders. An. Pediatr. (Barc.) 71, 489-494. doi: 10.1016/j.anpedi.2009.07.030

Carmichael, A. R., Sue-Ling, H. M., and Johnston, D. (2001). Quality of life after the Magenstrasse and mill procedure for morbid obesity. Obes. Surg. 11, 708-715. doi: 10.1381/09608920160558641

Chauvet-Gelinier, J. C., Roussot, A., Cottenet, J., Brindisi, M. C., Petit, J. M., Bonin, B., et al. (2019). Depression and obesity, data from a national administrative database study: geographic evidence for an epidemiological overlap. PLoS One 14:e210507. doi: 10.1371/journal.pone.0210507

Corp, I. (2016). IBM SPSS Statistics for Windows, Version 24.0. Armonk, NY: IBM Corp.

Dahm, J., Wong, D. N., and Ponsford, J. (2013). Validity of the depression anxiety stress scales in assessing depression and anxiety following traumatic brain injury. J. Affect. Disord. 151, 392-396. doi: 10.1016/j.jad.2013.06.011

Daumit, G. L., Clark, J. M., Steinwachs, D. M., Graham, C. M., Lehman, A., and Ford, D. E. (2003). Prevalence and correlates of obesity in a community sample of individuals with severe and persistent mental illness. J. Nerv. Ment. Dis. 191, 799-805. doi: 10.1097/01.nmd.0000100923.20188.2d

Diener, E., Emmons, R. A., Larsen, R. J., and Griffin, S. (1985). The Satisfaction with Life Scale. Journal of Personality Assessment. 49, 71-75. doi: 10.1207/ S15327752jpa4901_13

de Las Cuevas, C., Ramallo, Y., and Sanz, E. J. (2011). Are obesity and other physical comorbidities related to mental illness? Rev. Psiquiatr. Salud. Ment. 4, 119-125. doi: 10.1016/j.rpsm.2011.02.004

de Noronha, S. R., Campos, G. V., Abreu, A. R., de Souza, A. A., Chianca, D. A. Jr., and de Menezes, R. C. (2017). High fat diet induced-obesity facilitates anxiety-like behaviors due to GABAergic impairment within the dorsomedial hypothalamus in rats. Behav. Brain Res. 316, 38-46. doi: 10.1016/j. bbr.2016.08.042

de Wit, L. M., Fokkema, M., van Straten, A., Lamers, F., Cuijpers, P., and Penninx, B. W. (2010). Depressive and anxiety disorders and the association with obesity, physical, and social activities. Depress. Anxiety 27, 1057-1065. doi: $10.1002 /$ da. 20738

Dickerson, F. B., Brown, C. H., Kreyenbuhl, J. A., Fang, L., Goldberg, R. W., Wohlheiter, K., et al. (2006). Obesity among individuals with serious mental illness. Acta Psychiatr. Scand. 113, 306-313. doi: 10.1111/j.1600-0447.2005. 00637. $\mathrm{x}$

Esfahani, S. B., and Pal, S. (2018). Obesity, mental health, and sexual dysfunction: A critical review. Health Psychol. Open 5:2055102918786867. doi: $10.1177 / 2055102918786867$

EuroQol Group (1990). EuroQol-a new facility for the measurement of healthrelated quality of life. Health Policy 16, 199-208. doi: 10.1016/01688510(90)90421-9

EuroQol Group (2013). EQ-5D-3L User Guide. Available at: http://www.euroqol. org (Accessed September 9, 2021).

Fourrier, C., Bosch-Bouju, C., Boursereau, R., Sauvant, J., Aubert, A., Capuron, L., et al. (2019). Brain tumor necrosis factor-alpha mediates anxiety-like behavior in a mouse model of severe obesity. Brain Behav. Immun. 77, 25-36. doi: 10.1016/j.bbi.2018.11.316

Frezza, E. E., Shebani, K. O., and Wachtel, M. S. (2007). Laparoscopic gastric bypass for morbid obesity decreases bodily pain, improves physical functioning, and mental and general health in women. J. Laparoendosc. Adv. Surg. Tech. A 17, 440-447. doi: 10.1089/lap.2006.0069

Gariepy, G., Nitka, D., and Schmitz, N. (2010). The association between obesity and anxiety disorders in the population: a systematic review and metaanalysis. Int. J. Obes. 34, 407-419. doi: 10.1038/ijo.2009.252

Gracious, B. L., Cook, S. R., Meyer, A. E., Chirieac, M. C., Malhi, N., Fischetti, A. T., et al. (2010). Prevalence of overweight and obesity in adolescents with severe mental illness: a cross-sectional chart review. J. Clin. Psychiatry 71, 949-954. doi: 10.4088/JCP.09m05033gre

Haddadi, P., and Besharat, M. A. (2010). Resilience, vulnerability and mental health. Procd. Soc. Behv. 5, 639-642. doi: 10.1016/j.sbspro.2010.07.157

Halfon, N., Larson, K., and Slusser, W. (2013). Associations between obesity and comorbid mental health, developmental, and physical health conditions in a nationally representative sample of US children aged 10 to 17 . Acad. Pediatr. 13, 6-13. doi: 10.1016/j.acap.2012.10.007

Hayes, A. F. (2013). Methodology in the Social Sciences.Introduction to Mediation, Moderation, and Conditional Process Analysis: A Regression-Based Approach. United States: Guilford Press.

Hayes, A. F., Montoya, A. K., and Rockwood, N. J. (2017). The analysis of mechanisms and their contingencies: PROCESS versus structural equation modeling. Australas. Mark. J. 25, 76-81. doi: 10.1016/j.ausmj.2017.02.001

Henry, J. D., and Crawford, J. R. (2005). The short-form version of the depression anxiety stress scales (DASS-21): construct validity and normative data in a large non-clinical sample. Br. J. Clin. Psychol. 44, 227-239. doi: $10.1348 / 014466505 X 29657$

Holt, R. I., and Peveler, R. C. (2009). Obesity, serious mental illness and antipsychotic drugs. Diabetes Obes. Metab. 11, 665-679. doi: 10.1111/j. 1463-1326.2009.01038.x

Iacovides, A., and Siamouli, M. (2008). Comorbid mental and somatic disorders: an epidemiological perspective. Curr. Opin. Psychiatry 21, 417-421. doi: 10.1097/YCO.0b013e328303ba42

Jacobi, F., Hofler, M., Siegert, J., Mack, S., Gerschler, A., Scholl, L., et al. (2014). Twelve-month prevalence, comorbidity and correlates of mental disorders in Germany: the mental health module of the German health interview and examination survey for adults (DEGS1-MH). Int. J. Methods Psychiatr. Res. 23, 304-319. doi: 10.1002/mpr.1439

Jarvholm, K., Olbers, T., Marcus, C., Marild, S., Gronowitz, E., Friberg, P., et al. (2012). Short-term psychological outcomes in severely obese adolescents after bariatric surgery. Obesity 20, 318-323. doi: 10.1038/oby.2011.310

Kehoe, S., and O'Hare, L. (2010). The reliability and validity of the family affluence scale. Eff. Educ. 2, 155-164. doi: 10.1080/19415532.2010.524758

Keyes, C. L. M. (2005). Mental illness and/or mental health? Investigating the axioms of the complete state model of health. J. Consult. Clin. Psychol. 73, 539-548. doi: 10.1037/0022-006X.73.3.539

Kline, P. (2000). The Handbook of Psychological Testing. 2nd Edn. London, New York: Routledge.

Lovibond, P. F., and Lovibond, S. H. (1995). The structure of negative emotional states - comparison of the depression anxiety stress scales (DASS) with the Beck depression and anxiety inventories. Behav. Res. Ther. 33, 335-343. doi: 10.1016/0005-7967(94)00075-U

Lukat, J., Becker, E. S., Lavallee, K. L., van der Veld, W. M., and Margraf, J. (2016). Predictors of incidence, remission and relapse of Axis I mental disorders in young women: A transdiagnostic approach. Clin. Psychol. Psychother. 24, 322-331. doi: 10.1002/cpp.2026

Luppino, F. S., de Wit, L. M., Bouvy, P. F., Stijnen, T., Cuijpers, P., Penninx, B. W. J. H., et al. (2010). Overweight, obesity, and depression A systematic review and meta-analysis of longitudinal studies. Arch. Gen. Psychiatry 67, 220-229. doi: 10.1001/archgenpsychiatry.2010.2

Lyubomirsky, S., and Lepper, H. S. (1999). A measure of subjective happiness: preliminary reliability and construct validation. Soc. Indic. Res. 46, 137-155. doi: 10.1023/A:1006824100041

MacKinnon, D. P., Lockwood, C. M., and Williams, J. (2004). Confidence limits for the indirect effect: distribution of the product and resampling methods. Multivar. Behav. Res. 39, 99-128. doi: 10.1207/s15327906mbr3901_4

Maercker, A., Zhang, X. C., Gao, Z. H., Kochetkov, Y., Lu, S., Sang, Z. Q., et al. (2015). Personal value orientations as mediated predictors of mental health: A three-culture study of Chinese, Russian, and German university students. Int. J. Clin. Health Psychol. 15, 8-17. doi: 10.1016/j.ijchp.2014. 06.001

Margraf, J., and Schneider, S. (2017). Bochum Optimism and Mental Health (BOOM) Studies: Protocol for a Multi-National Longitudinal Research Program.

Margraf, J., Zhang, X. C., Lavallee, K. L., and Schneider, S. (2020). Longitudinal prediction of positive and negative mental health in Germany, Russia, and China. PLoS One. 15:e0234997. doi: 10.1371/journal.pone.0234997

McIntyre, R. S., Konarski, J. Z., Wilkins, K., Soczynska, J. K., and Kennedy, S. H. (2006). Obesity in bipolar disorder and major depressive disorder: results 
from a national community health survey on mental health and well-being. Can. J. Psychiatr. 51, 274-280. doi: 10.1177/070674370605100502

Michael, T., Zetsche, U., and Margraf, J. (2007). Epidemiology of anxiety disorders. Psychiatry 6, 136-142. doi: 10.1016/j.mppsy.2007.01.007

Myers, A., and Rosen, J. C. (1999). Obesity stigmatization and coping: relation to mental health symptoms, body image, and self-esteem. Int. J. Obes. Relat. Metab. Disord. 23, 221-230.

NCD Risk Factor Collaboration (2017). Worldwide trends in body-mass index, underweight, overweight, and obesity from 1975 to 2016: a pooled analysis of 2416 population-based measurement studies in 128.9 million children, adolescents, and adults. Lancet 390, 2627-2642. doi: 10.1016/S0140-6736(17)32129-3

Ng, F., Trauer, T., Dodd, S., Callaly, T., Campbell, S., and Berk, M. (2007). The validity of the 21-item version of the depression anxiety stress scales as a routine clinical outcome measure. Acta Neuropsychiatrica 19, 304-310. doi: $10.1111 / j .1601-5215.2007 .00217 . x$

Peduzzi, P., Concato, J., Kemper, E., Holford, T. R., and Feinstein, A. R. (1996). A simulation study of the number of events per variable in logistic regression analysis. J. Clin. Epidemiol. 49, 1373-1379.

Peng, Z., Zheng, Z., Han, H., Dong, C., Liang, J., Lu, J., et al. (2019). Imbalance in obesity and mental health among "little emperors" in China. PLoS One 14:e0207129. doi: 10.1371/journal.pone.0207129

Preacher, K. J., and Hayes, A. F. (2008). Asymptomatic and resampling strategies for assessing and comparing indirect effects in multiple mediator models. Behav. Res. Methods 40, 879-891. doi: 10.3758/BRM.40.3.879

Prisciandaro, J. J., and Roberts, J. E. (2009). A comparison of the predictive abilities of dimensional and categorical models of unipolar depression in the national comorbidity survey. Psychol. Med. 39, 1087-1096. doi: 10.1017/ S0033291708004522

Roberts, R. E., and Duong, H. T. (2016). Do anxiety disorders play a role in adolescent obesity? Ann. Behav. Med. 50, 613-621. doi: 10.1007/s12160-016-9786-8

Rucker, D. D., Preacher, K. J., Tormala, Z. L., and Petty, R. E. (2011). Mediation analysis in social psychology: current practices and new recommendations. Soc. Personal. Psychol. Compass 5, 359-371. doi: 10.1111/j.1751-9004.2011.00355.x

Schnohr, C. W., Kreiner, S., Due, E. P., Currie, C., Boyce, W., and Diderichsen, F. (2008). Differential item functioning of a family affluence scale: validation study on data from HBSC 2001/02. Soc. Indic. Res. 89, 79-95. doi: 10.1007/ s11205-007-9221-4

Schumacher, J., Leppert, K., Gunzelmann, T., Strauss, B., and Brahler, E. (2005). The resilience scale - A questionnaire to assess resilience as a personality characteristic. Zeitschrift Fur Klinische Psychol. Psychiatrie Und Psychother. 53, 16-39.

Scott, K. M., Bruffaerts, R., Simon, G. E., Alonso, J., Angermeyer, M., de Girolamo, G., et al. (2008). Obesity and mental disorders in the general population: results from the world mental health surveys. Int. J. Obes. 32, 192-200. doi: 10.1038/sj.ijo.0803701

Wagnild, G. M., and Young, H. M. (1993). Development and psychometric evaluation of the resilience scale. J. Nurs. Meas. 1, 165-178

Wittchen, H. U., Hofler, M., Gloster, A. T., Craske, M. G., and Beesdo, K. (2011). "Options and Dilemmas of Dimensional Measures for DSM-5: Which Types of Measures Fare Best in Predicting Course and Outcome?," in The Conceptual Evolution of DSM-5. Washington, 119-143.

WHO (2000). Cross-national comparisons of the prevalences and correlates of mental disorders. WHO International Consortium in Psychiatric Epidemiology. Bulletin of the World Health Organization 78, 413-426.

Conflict of Interest: The authors declare that the research was conducted in the absence of any commercial or financial relationships that could be construed as a potential conflict of interest.

Publisher's Note: All claims expressed in this article are solely those of the authors and do not necessarily represent those of their affiliated organizations, or those of the publisher, the editors and the reviewers. Any product that may be evaluated in this article, or claim that may be made by its manufacturer, is not guaranteed or endorsed by the publisher.

Copyright $\odot 2021$ Lavallee, Zhang, Schneider and Margraf. This is an open-access article distributed under the terms of the Creative Commons Attribution License (CC $B Y)$. The use, distribution or reproduction in other forums is permitted, provided the original author(s) and the copyright owner(s) are credited and that the original publication in this journal is cited, in accordance with accepted academic practice. No use, distribution or reproduction is permitted which does not comply with these terms. 